\title{
Avaliação da presença de risco para queda em idosos ${ }^{1}$
}

\author{
Evaluation of the risk of falls in the elderly
}

\section{Evaluación del riesgo de caídas en el anciano}

Tatiana Rocha Machado', Célida Juliana de Oliveira", Francisca Bertilia Chaves Costa'I", Thelma Leite de Araujo'V

${ }^{1}$ Pesquisa extraída da Monografia de término de curso de Graduação em Enfermagem pela Universidade Federal do Ceará.

' Enfermeira; Especialista em Enfermagem Clínica pela Universidade Estadual do Ceará e em Gestão Hospitalar pela Universidade Estadual Vale do Acaraú. Email: tatianarmachado@yahoo.com.br.

"Enfermeira; Mestre em Cuidados Clínicos pela Universidade Estadual do Ceará. Discente do curso de Doutorado em Enfermagem pela Universidade Federal do Ceará, Bolsista CAPES. Email: celidajuliana@yahoo.com.br

III Enfermeira; Mestre em Enfermagem pela Universidade Federal do Ceará. Email: bertilia_chaves@hotmail.com.

Iv Enfermeira; Doutora em Enfermagem; Docente da Universidade Federal do Ceará. Email: thelmaaraujo2003@yahoo.com.br.

\section{RESUMO}

As quedas são um importante fator causal para a dependência dos idosos, pois, estão relacionadas a um índice elevado de incapacidade e de mortalidade. Objetivou-se avaliar os fatores de risco para quedas presentes em um grupo de idosos de acordo com o diagnóstico de enfermagem "Risco para quedas" da NANDA (versão 2001/2002) e determinar o grau de susceptibilidade aumentado para quedas nos idosos segundo a "Escala de risco de quedas de Downton". Estudo descritivo realizado com 24 idosos no período de janeiro a julho de 2003 em um centro de saúde de Fortaleza/CE. Distribuiu-se os fatores em: maior freqüência; menor freqüência e as categorias não citadas. Fatores de risco mais citados: quedas (87,5\%); idade igual ou superior a 65 anos $(70,8 \%)$; dificuldades visuais (95,8\%); medicações (75\%); quarto não familiar (62,5\%) e ausência de antiderrapante $(95,8 \%)$. 0 processo de envelhecimento provoca inúmeras alterações no organismo humano, podendo levar o indivíduo a sofrer mudanças no seu cotidiano ao longo do tempo, como a necessidade de ajuda para realizar as atividades de vida diária. As quedas neste grupo etário podem ser consideradas como fatores significativos de alerta a fim de evitar seqüelas físicas e emocionais decorrentes desses eventos.

Descritores: Enfermagem geriátrica; Idoso; Acidentes por quedas.

\section{ABSTRACT}

Falls became an important factor for the dependence of the elderly, because they are related to a high rate of incapacity and mortality. The objective was to evaluate the risk factors of falls in a group of elderly according to the Nursing diagnose "Risk of falls" by NANDA (version 2001/2002) and determine the rate of falls that increased according to the "Downton's risk of falls Scale". Descriptive study conducted with 24 elderly, from January to July, at a health center in Fortaleza/CE. The factors were distributed in: biggest frequency; less frequency and categories nonquoted. Risk factors most often cited: falls (87.5\%); 65 years old or more (70.8\%); visual difficulties (95.8\%); medications (75\%); not familiar room (62.5\%) and absence of antiskid $(95.8 \%)$. The elderly process causes many changes in the human organism, such as suffering in the daily of elderly, because they need help to do their activities. The falls in this aged group can be considered as significant factors of alert in order to prevent physical and emotional sequels resulting from these events.

Descriptors: Geriatric nursing; Elderly; Accidental falls

\section{RESUMEN}

Las caídas son un factor causal importante para la dependencia del anciano, porque se relacionan con una proporción alta de incapacidad y mortalidad. El objetivo fue evaluar los factores de riesgo de caídas en un grupo del ancianos según el diagnóstico de Enfermería "Riesgo de caídas" del NANDA (versión 2001/2002) y determinar la proporción aumentada de caídas según el "Escala de riesgo de caídas de Downton". Estudio descriptivo realizado con 24 ancianos de enero a julio de 2003 en un centro de salud en Fortaleza-CE. Se distribuye en los factores: mayor frecuencia, menor frecuencia y las categorías non-mencionadas. Los factores de riesgo más frecuentemente citados: caídas $(87,5 \%)$, edad superior a 65 años $(70,8 \%)$, dificultades visuales $(95,8 \%)$, medicamentos (75\%); cuartos desconocidos $(62,5 \%)$ y falta de no deslizantes $(95,8 \%)$. El proceso de envejecimiento provoca muchos cambios en el organismo humano y puede llevar la persona a cambiar su vida cotidiana, como la necesidad de ayuda para la realización de actividades de la vida diaria. Las caídas en este grupo de edad pueden ser considerados como factores importantes de alerta para evitar efectos físicos y emocionales derivados de estos eventos.

Descriptores: Enfermería geriátrica; Ancianos; Accidentes por caídas. 


\section{NTRODUÇÃO}

Há cerca de 50 anos vêm ocorrendo mudanças na demografia populacional mundial. Especificamente no Brasil, em termos relativos, o grupo etário de pessoas com idade de 60 anos ou mais foi o que mais cresceu, principalmente a partir da década de $60^{(1)}$. Estatísticas demonstram 0 envelhecimento das populações como sendo conseqüência do desenvolvimento econômico e social, aliado à diminuição das taxas de natalidade e fecundidade, o aumento da expectativa de vida e a melhoria nas condições de infra-estrutura básica ${ }^{(2)}$.

Apesar do envelhecimento humano ser um processo gradual, irreversível e incontrolável de declínio das funções fisiológicas, ele não resulta, necessariamente, em incapacidade ${ }^{(3)}$, mas à medida que o indivíduo envelhece, as chances de sofrer lesões provocadas por acidentes aumentam ${ }^{(4)}$.

As quedas são importantes fatores causais para aumentar o nível de dependência do idoso, tornandose uma preocupação específica, já que podem afetar sua capacidade funcional por estar associada a modificações anatômicas atribuídas ao processo natural de envelhecimento e a diversas patologias ${ }^{(5)}$.

Neste contexto, as lesões causadas por acidentes estão em quinto lugar como causa de óbito em pessoas idosas, sendo que as quedas representam cerca de dois terços desses acidentes, tornando-se um dos principais previsores de morbimortalidade entre essa população(4), apesar de sua grande maioria ser evitável.

A segurança dos idosos deve ser motivo de preocupação para a sociedade, pois, para essas pessoas, as quedas podem ter repercussões desastrosas, uma vez que idosos com traumas têm perda na sua autonomia e aumento da sua dependência, refletindo em acréscimo de trabalho e estresse para o cuidador e familiares. Para se prevenirem desses acontecimentos, cuidadores e familiares devem se mobilizar em torno de cuidados especiais, adaptando o ambiente em que o idoso vive e tendo o cuidado de observar alguns itens de segurança, como o uso de calçados adequados, tapetes antiderrapantes e disposição da mobília em casa $^{(6)}$

A enfermeira deve procurar na sua prática com idosos identificar os fatores que influenciam na sua mobilidade, quer sejam eles físicos, psicológicos, sócio-culturais ou ambientais, a fim de que possam realizar suas atividades diárias sem riscos de sofrerem lesões por quedas. Prevenção de quedas é uma situação que envolve cuidadores, familiares e profissionais de saúde. Em relação à enfermagem, considera-se que o Risco para quedas representa um diagnóstico de enfermagem, isto é, uma situação que demanda intervenções de enfermagem ${ }^{(7)}$. A partir da identificação da clientela sujeita aos riscos para quedas, a enfermeira deve implementar ações visando a diminuição ou mesmo supressão da ocorrência do fenômeno.

Com o aumento da idade, alguns fatores biológicos, doenças e mesmo outras causas externas podem influenciar a fase de envelhecimento. A queda é uma dentre outras causas externas que trazem mais problemas aos idosos ${ }^{(8)}$. Para atuar de forma preventiva a estes eventos viu-se que seria essencial a utilização de um estudo que identificasse o grau de risco para quedas na população em questão, principalmente em relação ao equilíbrio, daí a escolha da Escala de risco de quedas de Downton ${ }^{(9)}$.

Com isso, objetivou-se identificar em idosos pertencentes a um grupo específico, a presença dos fatores de risco descritos pela North American Nursing Diagnosis Association (NANDA), versão dos anos 2001/2002 $2^{(7)}$, para o diagnóstico de enfermagem "Risco para quedas" e determinar o grau de susceptibilidade aumentado para quedas nos idosos, segundo a "Escala de risco de quedas de Downton"(9)

\section{METODOLOGI A}

Estudo descritivo, realizado no período de janeiro a julho de 2003 em um centro de saúde de um bairro de Fortaleza/CE. Seu desenvolvimento se deu com vinte e quatro idosos (idade igual ou superior a 60 anos), de ambos os sexos. Os mesmos participavam de um grupo de terceira idade, integrado ao referido centro de saúde, onde realizavam atividades de recreação e trabalhos artesanais, com freqüência semanal de aproximadamente trinta idosos.

Participaram do estudo todos aqueles que estavam presentes nas reuniões do grupo durante o período em que foi realizada a coleta de dados e que aceitaram participar do mesmo. Tendo em vista que a partir da avaliação do ambiente em que o idoso vive, a enfermeira pode saber se este idoso está ou não vulnerável a sofrer uma queda, neste estudo utilizou-se como instrumento de investigação, os fatores de risco apresentados para o diagnóstico de enfermagem "Risco para quedas"(7), juntamente com uma tabela adotada para pontuação de risco de quedas de Downton ${ }^{(9)}$.

O diagnóstico de enfermagem é um poderoso veículo que requer um pensamento crítico, analítico e acurado para comunicar adequadamente aos outros os fenômenos em que se envolve a Enfermagem, conceituado como um julgamento clínico sobre as respostas do indivíduo, da família ou da comunidade a problemas de saúde/processos vitais reais ou potenciais ${ }^{(7)}$.

A tabela de risco de quedas de Downton foi adotada neste estudo por enfocar alguns fatores presentes nas quedas a fim de poder determinar suas causas para corrigi-las. Nela estão agrupados os seguintes fatores: quedas anteriores, administração 
de medicamentos, déficit sensorial, estado mental e deambulação( ${ }^{(9)}$.

A análise dos dados foi realizada após tabulação dos achados de acordo com o grau de susceptibilidade aumentado para quedas encontrado no grupo avaliado.

Os idosos aceitaram participar da pesquisa depois de serem devidamente esclarecidos sobre os seus objetivos, assinando o termo de consentimento pós-informado, o qual lhes garantiu o anonimato e a possibilidade de se desligarem do estudo a qualquer momento se assim o desejassem, sem qualquer prejuízo para os mesmos. Além disso, o projeto foi aprovado pelo Comitê de Ética em Pesquisa da Universidade Federal do Ceará de acordo com a resolução 196/96 com o número de protocolo no $57 / 03$, sobre pesquisa envolvendo seres humanos ${ }^{(10)}$ e a instituição autorizou a realização do estudo em suas dependências.

\section{RESULTADOS E DISCUSSÃO}

Tendo em vista a importância de fatores de risco como faixa etária e sexo para a ocorrência de quedas, buscou-se inicialmente a relação existente entre o risco para quedas com essas variáveis. Posteriormente, de acordo com os objetivos propostos, foram avaliados conforme os fatores de risco encontrados para o diagnóstico de enfermagem "Risco para quedas"(7) e segundo a "Escala de risco para quedas" ${ }^{\prime 9)}$

Foram entrevistados vinte e quatro idosos, sendo 20 mulheres e quatro homens, com idade variando entre 61 e 86 anos, distribuídos em três categorias $^{(11)}$ : idoso jovem (60 a 74 anos), com dezenove pessoas $(79,1 \%)$; idoso (75 a 84 anos), quatro pessoas $(16,7 \%)$; e idoso velho (85 anos ou mais), com apenas uma pessoa $(4,2 \%)$.

O Brasil, de acordo com projeções intercensitárias realizadas pelo IBGE atualmente, tem aproximadamente 16 milhões de idosos residentes, representando mais de $8 \%$ da população total e sabe-se que até o ano de 2020, o país terá, em média, $16 \%$ de sua população na terceira idade(12). Percebe-se no grupo avaliado uma maior predominância do sexo feminino. Quando se avaliam grupos inseridos em programas de atenção à saúde, a presença de uma maioria de mulheres é marcante, pois elas procuram mais os serviços de saúde e grupos específicos. Essa predominância do sexo feminino é ainda mais notada em grupos de terceira idade $^{(11)}$.

O envelhecimento traz como conseqüências mais diretas a modificação do perfil epidemiológico brasileiro, com crescimento significativo das doenças denominadas crônicas não transmissíveis. Entre elas, a hipertensão ocupa um efetivo espaço no hall das doenças crônicas comuns entre os mais velhos. Não que esta se configure como uma doença exclusiva desse grupo, mas é nítida sua maior incidência nessa clientela, onde no Brasil, em mais de $50 \%$ dos idosos, encontra-se com grande freqüência a hipertensão arterial $^{(13)}$. Por conta disso, considerouse importante caracterizar o grupo estudado de acordo com as doenças mais presentes.

Foram citadas pelos idosos as doenças diagnosticadas por médicos e para as quais fazem tratamento, estando entre elas a hipertensão arterial, relatada por 18 idosos $(75,0 \%)$, sendo a mais freqüente nesse grupo; osteoporose, com três pessoas; labirintite, apresentada por um idoso e diabetes mellitus, também com uma pessoa.

Apesar das doenças características do envelhecimento, os idosos valorizam a procura do bem viver, demonstram comportamentos adaptativos e possibilidade de maiores diversões e distrações, como a permanência com a família, amigos e a participação em grupos $^{(14)}$. A enfermagem tem um grande potencial para trabalhar com grupos e os idosos formam uma parcela da população que aproveita muito bem o convívio em grupos de autoajuda.

Avaliar a susceptibilidade para a ocorrência de queda nos idosos é uma opção viável e adequada por se acreditar que os diagnósticos representam o foco do cuidado de enfermagem.

Um diagnóstico de enfermagem pode ser vigente, também chamado de atual, de risco, do tipo de saúde ou de síndrome. O diagnóstico de enfermagem de risco, modalidade utilizada neste estudo, descreve um julgamento clínico em que um indivíduo/grupo está mais vulnerável ao desenvolvimento de um problema do que outros na mesma situação ou em situação $\operatorname{similar}^{(7)}$.

O processo de elaboração de um diagnóstico de enfermagem é difícil porque as enfermeiras tentam diagnosticar respostas humanas, as quais são exclusivas, pois cada ser humano é único em sua essência, vive em permanentes mudanças, portanto, as tentativas de classificação dessas respostas têm sido trabalhosas ${ }^{(15)}$. Ressalta-se, ainda, que os conhecimentos da enfermeira sobre os sinais e sintomas para os diagnósticos vigentes, os fatores de risco e as possíveis complicações direcionam a coleta de dados. A seguir serão apresentadas, em forma de tabela, as categorias de fatores de risco encontradas segundo NANDA 2001/2002:

Na Tabela 1 estão apresentadas as 28 categorias de fatores de risco do Diagnóstico de Enfermagem Risco para quedas, segundo a NANDA 2001/2002(7). Para sua análise, distribuíram-se os fatores encontrados da seguinte forma: a) Maior freqüência das categorias; b) Menor freqüência e c) As categorias que não foram citadas. Esta divisão seguiu-se ainda de acordo com o sexo (feminino e masculino e o que foi comum a ambos). 
Tabela 1: Distribuição da presença dos fatores de risco do diagnóstico "Risco para quedas" segundo NANDA 2001/2002, por sexo. Fortaleza/CE, 2003.

\begin{tabular}{|c|c|c|c|c|c|}
\hline \multirow{4}{*}{ Categorias } & \multirow{4}{*}{ Fatores de risco } & \multicolumn{4}{|c|}{ Sexo } \\
\hline & & \multirow{2}{*}{\multicolumn{2}{|c|}{$\begin{array}{c}\text { Masculino } \\
\mathrm{n}=05\end{array}$}} & \multirow{2}{*}{\multicolumn{2}{|c|}{$\begin{array}{c}\text { Feminino } \\
n=19\end{array}$}} \\
\hline & & & & & \\
\hline & & FA & $\%$ & FA & $\%$ \\
\hline \multirow{12}{*}{ Em adultos } & História de quedas & 4 & 80 & 17 & 89,5 \\
\hline & Uso de cadeiras de rodas & - & - & 1 & 5,2 \\
\hline & Idade $\geq 65$ anos & 5 & 100 & 12 & 63,1 \\
\hline & Morar sozinho & 1 & 20 & 2 & 10,5 \\
\hline & Uso de artefato de auxílio (bengala, etc.) & - & - & 5 & 26,3 \\
\hline & Doença vascular & - & - & 3 & 15,8 \\
\hline & Fadiga, mobilidade limitada & - & - & 3 & 15,8 \\
\hline & Incontinência urinária & 1 & 20 & 4 & 21 \\
\hline & Problemas nos pés & - & - & 2 & 10,5 \\
\hline & Mobilidade física prejudicada & - & - & 5 & 26,3 \\
\hline & Equilíbrio prejudicado & - & - & 1 & 5,3 \\
\hline & Dificuldade na marcha & 1 & 20 & 5 & 26,3 \\
\hline \multirow{6}{*}{ Fisiológicos } & Presença de doença aguda & 2 & 40 & 5 & 26,3 \\
\hline & Condições pós-operatórias & - & - & 1 & 5,2 \\
\hline & Dificuldades visuais & 5 & 100 & 18 & 94,7 \\
\hline & Dificuldades auditivas & - & - & 2 & 10,5 \\
\hline & Hipotensão ortostática & - & - & 2 & 10,5 \\
\hline & Ausência de sono & 1 & 20 & 7 & 36,8 \\
\hline Cognitivos & Estado mental diminuído & - & - & 1 & 5,2 \\
\hline \multirow{2}{*}{ Medicações } & Anti-hipertensivo & 4 & 80 & 14 & 73,7 \\
\hline & Outros & - & - & 8 & 42,1 \\
\hline \multirow{4}{*}{ Ambientais } & Tapetes pelo chão & 2 & 20 & 6 & 31,6 \\
\hline & Ambiente com móveis em excesso & - & - & 5 & 26,3 \\
\hline & Quarto não familiar ou pouco iluminado & 4 & 80 & 11 & 57,9 \\
\hline & Ausência de material antiderrapante & 5 & 100 & 18 & 94,7 \\
\hline
\end{tabular}

FA: Freqüência absoluta de idosos

Os fatores de risco com maior freqüência para ambos os sexos foram: história de quedas $(87,5 \%)$; idade igual ou superior a 65 anos $(70,8 \%)$; dificuldades visuais $(95,8 \%)$; uso de medicações $(75 \%)$; quarto não familiar ou pouco iluminado $(62,5 \%)$ e ausência de material antiderrapante $(95,8 \%)$.

Analisando em separado apenas os idosos do sexo masculino, foram encontrados os mesmos fatores, entretanto, quando se refere ao uso de medicações, tem-se que quase a totalidade desses idosos utilizava especificamente anti-hipertensivos, enquanto as mulheres utilizavam outros medicamentos, além destes.

Os fatores de risco menos presentes em ambos os sexos foram: morar sozinho $(12,5 \%)$ e incontinência urinária $(20,8 \%)$. Alguns fatores de risco relacionados pela NANDA ${ }^{(7)}$ não foram identificados nos idosos: uso de cadeira de rodas; prótese de membro inferior; artrite e uso de álcool.

Analisando os idosos do sexo masculino, não foram citados: uso de cadeira de rodas; prótese de membro inferior; uso de bengala ou muleta; doença vascular; fadiga; problemas nos pés; mobilidade física prejudicada; equilíbrio prejudicado; condições pós-operatórias; dificuldades auditivas; artrite; hipotensão ortostática; estado mental diminuído; uso de medicamentos que não fossem anti-hipertensivos; uso de álcool; ambiente com móveis em excesso. Já entre as idosas, apenas três fatores de risco não foram encontrados: prótese de membro inferior; artrite e uso de álcool.

As quedas em indivíduos acima dos 60 anos são tão freqüentes que há muito tempo são aceitas como efeitos "naturais" do envelhecimento. Tal observação repousa no fato que pelo menos $30 \%$ dos idosos no Brasil sofrem um episódio de queda por ano, sendo que as mulheres têm uma freqüência de quedas um pouco mais elevada que os homens da mesma faixa etária. Os idosos na faixa entre 75 e 84 anos que necessitam de auxílio em algumas atividades da vida diária têm probabilidade de sofrer queda aumentada em 14 vezes $^{(16)}$.

A enfermagem deve atuar com medidas preventivas para que essas estatísticas possam ser modificadas, detectar os fatores de risco físicos e ambientais, a fim de modificá-los ou adaptá-los, diminuindo assim o grau de susceptibilidade para 
quedas presente na população idosa.

Em estudo realizado no ano de 2000, os autores que investigavam a história de quedas (local de ocorrência, causas e conseqüências) referidas por idosos da cidade de Ribeirão Preto, identificaram que $54 \%$ das quedas tinham como causa principal um ambiente inadequado para o idoso(17).

Os profissionais ao orientarem o idoso, devem apresentar as possibilidades de risco a que estes estão sujeitos, encorajar a adaptação do ambiente, solicitando sua participação. Isto faz com que estes percebam as necessidades e façam opções para tornar o ambiente seguro e confortável, principalmente, àqueles que apresentam dificuldade para andar, utilizam bengala, muletas, cadeira de rodas e necessitam de pessoas para transferi-los de um lado para o outro. É importante lembrar que o espaço em que o idoso vive e se movimenta deve ficar mais livre possível de móveis e utensílios que possam causar ferimentos ${ }^{(5)}$.

Tabela 2: Distribuição dos idosos de acordo com as pontuações encontradas segundo sexo e a "Escala de risco de quedas de Downton". Fortaleza/CE, 2003.

\begin{tabular}{|c|c|c|c|c|}
\hline \multirow{2}{*}{ Fatores de risco } & \multicolumn{4}{|c|}{ Sexo } \\
\cline { 2 - 5 } & \multicolumn{2}{|c|}{ Masculino } & \multicolumn{2}{c|}{ Feminino } \\
\cline { 2 - 5 } & FA & Pontuação & FA & Pontuação \\
\hline Quedas anteriores & 3 & 1 & 9 & 1 \\
\hline Dificuldade visual & 1 & 1 & 5 & 1 \\
\hline Dificuldade auditiva & - & - & 1 & 1 \\
\hline Uso de tranqüilizantes/ sedativos & 1 & 1 & 3 & 1 \\
\hline Uso de hipotensor & 3 & 1 & 14 & 1 \\
\hline Estado mental confuso & - & - & 1 & 1 \\
\hline Deambulação insegura com ajuda (bengala) & 1 & 1 & 3 & 1 \\
\hline Dificuldade na marcha & 1 & 1 & 3 & 1 \\
\hline
\end{tabular}

FA: Freqüência absoluta de idosos

É preciso estar claro que a queda é um evento real na vida dos idosos e traz a eles muitas conseqüências, às vezes irreparáveis, e que a incapacidade para realizar atividades da vida diária pode trazer, a longo prazo, conseqüências não só para os idosos mas também para a família e para os serviços de saúde, que precisam se mobilizar para o tratamento e recuperação do idoso. Para minimizar as conseqüências e reduzir danos aos idosos provocados pelas quedas, torna-se necessário investir em campanhas que envolvam a prevenção destes acidentes entre os mesmos. Faz-se necessário manter um meio de comunicação eficaz que facilite a compreensão e estimule sua prática e que torne o idoso e sua família participantes ativos do processo de promoção de sua saúde ${ }^{(18)}$.

Com a aplicação da "Escala de risco de quedas"(9) foi calculado o grau de suscetibilidade para quedas, no grupo avaliado. No estudo aplicou-se a escala a partir dos fatores de risco para o diagnóstico de enfermagem "Risco para quedas"(7).

Foram distribuídas as categorias de pontuações encontradas de acordo com o sexo. Totalizaram dez categorias associadas e duas categorias isoladas.

Para o sexo masculino, foram encontrados dois grupos de fatores associados. A pontuação variou de um a quatro pontos:

- Quedas anteriores + Dificuldade na marcha + Dificuldade visual + Uso de hipotensores (totalizando 4 pontos): um idoso.

- Quedas anteriores + Dificuldade visual + Uso de hipotensores ( 3 pontos): três idosos.
O único fator encontrado isoladamente foi a dificuldade visual presente em um idoso.

Para o sexo feminino foram encontrados dez fatores associados e um fator isolado. Os grupos de fatores associados que apresentaram maior pontuação foram:

- Quedas anteriores + Dificuldade visual + Uso de hipotensores + Uso de tranqüilizantes/sedativos + Deambulação insegura com ajuda (bengala) + Estado mental confuso (seis pontos): Uma idosa.

- Quedas anteriores + Dificuldade visual + Dificuldade auditiva + Deambulação insegura com ajuda (bengala) + Uso de hipotensores (cinco pontos): Duas idosas.

A menor pontuação encontrada foi de um ponto (uma idosa) com o fator "Quedas anteriores". Apenas uma categoria não foi identificada de forma isolada nas idosas avaliadas: dificuldade visual.

Os fatores ambientais são os que proporcionam maior risco de queda e, quando associados a características físicas dos indivíduos, são ainda mais agravantes. Quando existe o comprometimento, as atividades físicas significam não somente prejuízo no equilíbrio em si, que seria o fator primordial no comprometimento das atividades instrumentais, como também limitações da força muscular, da mobilidade e da marcha. Por isso, nas situações de assistência a saúde, estes fatores devem sempre ser avaliados $^{(18)}$.

Um estudo realizado com 137 idosos que sofreram pelo menos um evento de queda no ano de 
2004 pretendeu identificar variáveis contribuintes para a alta freqüência de quedas entre os idosos a partir de diagnósticos de enfermagem. Foi verificada associação entre a ocorrência do evento e a perda de equilíbrio, a pressão arterial elevada e fraqueza muscular, além de que os idosos que faziam uso de quatro ou mais drogas apresentaram história de quedas em maiores proporções ${ }^{(19)}$. Tais fatos corroboram os achados no presente estudo, já que as maiores pontuações encontradas em nossos idosos apresentavam associação de pelo menos dois fatores semelhantes ao do estudo anteriormente comentado.

Dentre as alterações fisiológicas mais observadas no estudo encontram-se as oculares e as auditivas, comuns nos idosos, as quais somadas às alterações relacionadas ao envelhecimento acabam reduzindo sua autonomia e independência, ocasionando prejuízos na sua qualidade de vida.

O envelhecimento ocular experimenta alterações que podem afetar adversamente a visão. Qualquer alteração nessa função pode aumentar o risco de quedas caso algum objeto sobre o chão não seja visualmente detectado. O envelhecimento também é acompanhado por presbiacusia, uma perda neurosensorial da audição, que afeta a habilidade de detectar ruídos ${ }^{(20)}$.

É sabido que o risco para quedas aumenta proporcionalmente com o número de fatores de risco, por ser um evento que dificilmente é resultado de um fator isolado. Daí a importância da enfermagem agir preventivamente em relação às quedas em idosos, procurando atuar sobre a maior quantidade possível desses fatores.

\section{CONCLUSÃO}

O processo de envelhecimento provoca inúmeras alterações no organismo humano, podendo levar o indivíduo a sofrer mudanças no seu cotidiano, como por exemplo, aumento da possibilidade de sofrer quedas que levam os idosos a tornarem-se mais dependentes de ajuda para realizar as atividades de vida diária.

Observou-se no estudo que os dois instrumentais utilizados para abordagem com os idosos que as quedas podem ser consideradas como fatores significativos de alerta com a população idosa, a fim de evitar seqüelas físicas e até mesmo emocionais decorrentes desses eventos.

No estudo surgiram questionamentos sobre o ambiente em que o idoso vive para identificar se existem riscos para quedas envolvidos. Observou-se que foram relatados pelos idosos alguns problemas com o ambiente, os quais podem propiciar as quedas. No entanto, acredita-se que este ponto seja um fator limitante para o estudo, pois, não foram realizadas visitas às residências dos idosos para confirmação das falas, assim, como envolver também os cuidadores neste processo.
Todos os vinte e quatro idosos apresentaram fatores de risco para o diagnóstico de enfermagem "Risco para quedas". Um dos fatores mais presentes foi a idade superior a 65 anos, o que já torna o idoso susceptível a quedas independente da presença de outros fatores de risco. Além deste, foram identificados diversos outros fatores interdependentes, fazendo aumentar o grau de susceptibilidade para sofrer quedas demonstrando a importância da avaliação de enfermagem como um todo.

Com isso, pode-se sugerir que os profissionais de saúde devem orientar os idosos a adotar atitudes saudáveis que podem prevenir as quedas. Algumas dessas atitudes podem ser elencadas como: realização de atividade física regular, consultas para avaliação dos níveis de pressão arterial e uso de medicamentos e os cuidados com o ambiente em que o idoso vive.

O idoso necessita de atenção, cuidado, incentivo e valorização da sua história de vida. O enfermeiro, como membro da equipe de saúde deve desenvolver, aprimorar e socializar cuidados clínicos e estratégias para melhor atendimento às necessidades bio-psicosociais dessa faixa etária, onde o envelhecimento saudável não deve fazer parte somente das preocupações do setor saúde; deve ser incluída como prioridade na agenda social do país.

\section{REFERÊNCIAS}

1. Mendes MRSS, Gusmão JL, Faro ACM, Leite RCBO. A situação social do idoso no Brasil: uma breve consideração. Acta paul. enferm. 2005; 18(4):422-6.

2. Fardo VM, Creutzberg M, Silva MCS. Qualidade de vida de idosos hospitalizados: um estudo preliminar. Revista Nursing. 2005; 86(8): 314-9.

3. Lueckenotte A. Avaliação da enfermagem para a pessoa idosa. In: Lueckenotte A. Avaliação em gerontologia. 3rd ed. Rio de Janeiro: Reichmann \& Affonso; 2002. p. 1-3.

4. Felten BS, Vickey PG, Mangin EJ, Purvis G, RossKerr JC, Vontz MJ. Quedas e sua prevenção. In: Felten BS, Vickey PG, Mangin EJ, Purvis G, Ross-Kerr JC, Vontz MJ. Geriatria e gerontologia. Rio de Janeiro: Reichmann \& Autores; 2005. p. 515-21. (vol. 2).

5. Silva TM, Nakatani AYK, Souza ACS, Lima MCS. A vulnerabilidade do idoso para as quedas: análise dos incidentes críticos. Rev. Eletr. Enf. [Internet]. 2007 [cited 2009 feb 12];9(1):64-78 Available from: http://www.fen. ufg.br/revista/v9/n1/v9n1a05.htm.

6. Coutinho ESF, Silva SD. Uso de medicamentos como fator de risco para fratura grave decorrente de queda em idosos. Cad. Saúde Pública. 2002; 18(5): 1359-66.

7. North American Nursing Diagnosis Association. Diagnósticos de Enfermagem: Definições e classificação - 2001/2002. Porto Alegre: Artmed; 
2002. $184 p$.

8. Gonçalves LG, Vieira, ST, Siqueira, FV. Prevalência de quedas em idosos asilados do município de Rio Grande, RS. Rev. Saúde Públ. [Internet]. 2008 [cited 2009 mar 05];42(5):938-45. Available from: http://www. scielo.br/pdf/rsp/v42n5/6845.pdf.

9. Downton JH. Falls in the elderly. Londres: Edward Arnold; 1993.

10. Ministério da Saúde; Conselho Nacional de Saúde. Resolução No 196/96 - Normas regulamentadoras de pesquisa envolvendo seres humanos. Brasília (Brasil): Ministério da Saúde; 1996.

11. Vieira MDCM. Velhice feminina no asilo: do imaginário ao real [thesis]. Fortaleza: Universidade Federal do Ceará. Doutorado em Enfermagem. Departamento de Enfermagem; 2001. 162 p.

12. Instituto Brasileiro de Geografia e Estatística [Internet]. Brasília: Ministério do Planejamento, Orçamento e Gestão (BR) [cited 2009 feb 27]. Pesquisa Nacional por Amostra de Domicílios. Síntese de Indicadores 2005. Available from: http://www.ibge.gov.br/home/estatistica/populacao/t rabalhoerendimento/pnad2005/default. shtm.

13. Sociedade Brasileira de Hipertensão [Internet]. São Paulo: Sociedade Brasileira de Cardiologia (BR) [update 2007 sep 03, cited 2009 feb 12]. V Diretrizes Brasileiras de Hipertensão Arterial. Available from: http://www.sbn.org.br/Diretrizes/V_Diretrizes_Brasil eiras_de_Hipertensao_Arterial.pdf.

14. Oliveira TC, Melo EM, Almeida DT, Araujo TL. Envelhecimento e qualidade de vida: trabalhando com um grupo de idosos. In: Forte BP, Jorge MSB, Soares E. Complexidade e diversidade do conhecimento em saúde: estimulando uma cultura de intervenções multiprofissionais. Fortaleza: EUFC; 2002. p. 41-51.

15. Carpenito-Moyet LJ. Diagnósticos de enfermagem. Aplicação à prática clínica. 10 ed. Porto Alegre: Artmed; 2005.

16. Pereira SRM, Buksman S, Perracini M, Py L, Barreto KML, Leite VMM. Projeto Diretrizes: Quedas em Idosos. São Paulo: Sociedade Brasileira de Geriatria e Gerontologia. 2001. 9p.

17. Fabrício SCC, Rodrigues RAP, Costa Junior ML. Causas e conseqüências de quedas de idosos atendidos em hospital público. Rev. Saúde Públ. 2004; 38(1): 93-9.

18. Lopes MCL, Violin MR, Lavagnoli AP, Marcon SS. Fatores desencadeantes de quedas no domicílio em uma comunidade de idosos. Cogitare Enferm. 2007; 12(4): 472-7.

19. Moreira MD, Costa AR, Felipe LR, Caldas CP. The association between nursing diagnoses and the occurrence of falls observed among eldery individuals assisted in an outpatient facility. Rev Latino-am Enfermagem. 2007; 15(2): 311-7.

20. Smeltzer SC, Bare BG. Enfermagem médico- cirúrgica. 10 ed. Rio de Janeiro: Guanabara Koogan; 2005.

Artigo recebido em 01.02.08

Aprovado para publicação em 31.03.09. 